\title{
Hersiening van die Fisikawoordeboek
}

Na die verskyning van die tweetalige Fisikawoordeboek in 1977 is tot 1989, met behulp van 'n aantal medewerkers wat jaarliks tydens die Suid-Afrikaanse Instituut vir Fisika (SAIF) se jaarvergadering bymekaar gekom het, 700 terme versamel.

Teen die einde van 1990 is in samewerking met die SA Akademie vir Wetenskap en Kuns en die Nasionale Vakterminologiediens (NVD) 'n nuwe komitee saamgestel met dr. D.F. Louw as voltydse Projekleier.

'n Redaksiekomitee bestaande uit vyf lede is benoem. Verder het 'n aantal vakkundiges hulle die benoeming as korresponderende lede laat welgeval. Die NVD tree as sekretariaat op.
Die projek sal in twee fases hanteer word. Fase A behels die behandeling van die beskikbare data, d.w.s. die terme uit die Fisikawoordeboek en die SAIF se aanvullingslys. In fase B sal daar aandag aan nuwe terme, terme van die vinnig ontwikkelende subvakdissiplines, gegee word.

Volgens die NVD se opdrag behels fase A "die verwerking, bywerk en evaluering van aanvullende (hoofsaaklik die SAIF se) termlyste; die redigering, evaluering, terminologiese versorging en afronding van die woordeboek in sy geheel, en die navorsing oor en benoeming van terme waar nodig. Die leksikografiese en redaksionele metodiek van die NVD (soos in hul nuwe handleiding aangedui) moet gevolg word". 


\section{Indeks: Jaargang 10}

(Die uitgawe en bladsynommer volg na die titel van die bydrae)

Baker, C. en A. Oldewage. Morfologie van die kliere geassosieerd met die labiaalstekels van Argulus japonicus Thiele, 1900 (Crustacea: Branchiura): 1, 52.

Basson, W.D. Die chemikus as entrepreneur: 3, 144.

Bekker, A. en J.J.J. Roux. Parameterryke onvoorwaardelike verdelings met 'n stogastiese parameter in die voorwaardelike gammaverdeling: 4, 155.

Bergh, J.J., J.C. Breytenbach en S. van Dyk. In vitro antimikrobiese aktiwiteit en in vivo-toksisiteit van die afbraakprodukte van trimetoprim: 4, 153.

Bezuidenhout, H. en G.J. Bredenkamp. Die plantgemeenskappe van die Ba-landtipe in die Mooirivieropvanggebied, Transvaal: 2,85 .

Bezuidenhout, H. en G.J. Bredenkamp. Plantegroeiklassifikasie van die A-landtipe van die Mooirivieropvanggebied, Transvaal: 1, 4.

Bornman, M.S., D. du Toit, M.H. Fourie, C. de VilliersSmith, E.L. Kok en D.J. du Plessis. Kliniese bevindinge by pasiënte met losgelate siliêre klossies (LSKs) in semen: 1, 58.

Bornman, M.S., L. Grobler, M. Mahomed, D. Boomker, G.W. Schulenburg, S. Reif en H.H. CreweBrown: Trichomonas vaginalis en manlike infertiliteit: $1,58$.

Botha, A. en F.C. Botha. Verandering in pirofosfaat: Dfruktose 6-fosfaat-1-fosfotransferase-aktiwiteit en -konsentrasie gedurende kieming van $C$. lanatus-saadlobbe: $1,44$.

Botha, F.C. Regulering van koolhidraatmetabolisme in Selenastrum minutum: $1,48$.

Botha, L. Suid-Afrika se seevisbronne: 4, 151 .

Bredenkamp, P. Kwaliteitsonderwys in die natuurwetenskappe: 3, 98.

De Beer, A.S. en J.D. van Wyk. Oordrag van energie in nielineêre elektriese netwerke - 'n moderne beskouing van drywing: 4, 160.

De Wet, E.H., J.M.C. Oosthuizen, H.C. Barnard, H.G. Luus en M.S. Bornman. Streshormone en fisieke aktiwiteit: 2, 93.

Du Pisani, A.L. Die aanwending van 'n eenvoudige grondwaterbalans om droogte in natuurlike veld te karakteriseer: 1, 26.

Du Plessis, S. en F.C. Botha. Die uiting van PFP-aktiwiteit in selkulture van Phaseolus vulgaris: 1, 49.

Du Preez, H.H. Die effek van voeding op die roetinesuurstofverbruik van die grasgarnaal Penaeus monodon: 1, 54.

Du Toit, D., M.S. Bornman en D.J. du Plessis. Adenosientrifosfaatkonsentrasie van menslike sperme en spermmotiliteit: 1, 57.

Du Toit, D., M.S. Bornman en D.J. du Plessis. Die moontlike rol van sialiensuur in manlike infertiliteit: 1 , 58.

Esterhuizen, A.D., F. le R. Fourie, J.V. van der Merwe en H. Hattingh. Voorlopige resultate in die gebruik van mikromanipulasie in 'n diermodel: 1, 61 .
Esterhuizen, A.D., C. Huyser en F. le R. Fourie. Die voorkoms en invloed van meervoudige oösiete op die uitkoms van 'n in vitro-bevrugtingsprogram: 1, 61.

Esterhuizen, H.J., L.C. Holtzhausen en E. Swart. Die invloed van drie kruisbestuiwers op die saadinhoud van Minneola tangelo (Citrus paradisi x C. reticulata): 4 , 169.

Esterhuizen, H.J., L.C. Holtzhausen en E. Swart. Ondersoek na vruggroei van Minneola tangelo (Citrus paradisa x $C$. reticulata): 2,80 .

Ferreira, D.I. Biotegnologie - 'n Nuwe benadering in planteteling: 1, 18.

Ferreira, D.I. Biotegnologieterme: 1, 36.

Fourie, F. le R., C. Huyser, D. Visser, A. Esterhuizen en E. Peters. Die invloed van endotoksienbesmetting op die uitkoms van 'n in vitro-bevrugtingsprogratu: 1. 62.

Fourie, F. le R. en J.V. van der Merwe. Lic :oukoms van Profase 1-oösiete en die invloed van verlengde inkuberingstyd op embrio-ontwikkeling: 1, 60 .

Fourie, J.H. Die internasionale bemarking van tegnologie: 3, 145.

Geertsema, A. Die oorbrugging tussen chemie en chemiese ingenieurswese: $3,146$.

Groenewald, E.G. en A.J. van der Westhuizen. Pogings vir die daarstelling van morfogenese uit kallus van Phaseolus vulgaris L: 1, 47.

Gründlingh, M.L. Draaikolke suid van die Mosambiekkanaal: 3, 140.

Henning, P.E., J.G.C. Small en A.J. van der Westhuizen. Proteien- en iso-ensiemprofiele van Strelitzia-spesies: I, 42.

Heydorn, A.E.F. Die bewaring van ons kusomgewing: 4, 150.

Holtzhausen, L.C. en L.A. Genis. Woordeboek vir Tuinboukunde (Ingeslote Plantname): 1, 36.

Huyser, C., D. du Toit, F. le R. Fourie en M.S. Bornman. Sialiensuur in follikulêre vog: 1,60 .

Huyser, C. en F. le R. Fourie. Hormonale bepalings van follikulêre vog deur middel van fluoro-immunometrie: 1,60 .

Kemp, K. en J.G.C. Small. Die invloed van suurstofspanning op mitochondriale proteïne van emrionale assies van Pisum sativum L.: 1, 45.

Knight, E. en A. Avenant-Oldewage. Die anatomie en morfologie van die spysverteringskanaal van Chonopeltis australis (Crustacea: Branchiura): 1, 52.

Krüger, G.H.J., E.G. Groenewald en P.J. Botes. Die opsporing van prostaglandien F2a in prokariotiese organismes met behulp van radio-immuno-essaïering: 1,50 .

Kruger, M., J.H.J. van Vuren en H.H. du Preez. 'n Voorlopige ondersoek na die moontlike swaarmetaalbesoedeling van die Olifantsrivier in die Nasionale Krugerwildtuin: 1, 51.

Malherbe, F.E. Die rol van die Stigting vir Onderwys, Wetenskap en Tegnologie: 3, 144. 
Meij, H.S. Die bloed-brein-skans: 3, 100.

Noble, R.G. Chemiese afval en die omgewingskrisis: 3, 145.

Oosthuizen, S., F.H. van der Bank en J.T. Ferreira. 'n Biochemiese en morfologiese ondersoek van Tilapia guinasana, $T$. rendalli en $T$. sparrmanii: 1, 56.

Pieterse, A.J.H. Fitoplankton in gesuiwerde Vaalrivierwater: 1,41 .

Potgieter, G.P. en F.C. Botha. 'n Model vir kiemingsbeheer van Citrullus lanatus-sade: 1, 43.

Reinecke, A.J. Omgewingstoksikologie - 'n nuwe uitdaging: 2, 66.

Reinecke, C.J. Universiteitswese in die toekomstige SuidAfrika: 3, 143.

Saayman, R. Die fisika van motorbotsings en padverkeersveiligheid: 3,112 .

Sander, C.L. Die voorsiening van opgeleide chemici realiteite en probleme: $3,142$.

Schmidt, A.R. en A.J.H. Pieterse. Die invloed van mangaan en koper op die groei van Cosmarium- en Cyclotella-spesies uit die Vaalrivier: 1, 40.

Scholtz, M.M. en C.Z. Roux. Evaluasie van bestaande seleksiekriteria vir beeste in terme van totale kuddedoeltreffendheid: 3, 106.

Schultz, C. en J.G.C. Small. Hoë temperatuurinhibering van slaaisaadontkieming: 1, 46.

Schutte, H.J. Primitiewe elemente vir kommutatiewe ringuitbreidings: $2,67$.

Slabber, C.F. Gesondheid in Afrika: 1, 30.

Steenkamp, V.E., H.H. du Preez en H.J. Schoonbee. 'n Ekologiese ondersoek na die bio-akkumulering van sink, yster en mangaan in die varswaterkrap Potamonautes warreni (Calman): 1, 54.

Strassberger, F.G.W., P.P.C. Nel en M.M. Nel. Die ligen transmissie-elektronmikroskopiese struktuur van die byniere van 'n nie-dragtige, nie-sogende Afrikaolifantkoei (Loxodonta africana): 1, 59.

Strydom, B.W. Is daar vordering met die benutting van biologiese stikstofbinding: 1,2 .
Swanepoel, J.H. Bydraes van Darwin se voorgangers tot die ewolusieteorie: 1, 11.

Trautmann, I.A. en J.H. Visser. Protoplast isolering van Parthenium argentatum (Guayule): 1, 46.

Van Aardt, W.J. Die invloed van oefening op hemosianienfunksie: 1, 57.

Van Aardt, W.J. 'n Plastiek Cartesiese duiker as respirometer: $4,169$.

Van Blerk, J.M. Omgewingsfaktore en fitoplanktonproduksie in die Vaalrivier by Balkfontein: 1, 40.

Van der Walt, J.J.A., H.J.T. Venter en R. Verhoeven. Erodium incamatum word oorgeplaas na die genus Pelargonium: 1, 51.

Van der Westhuizen, A.J. en S. du Plessis. Lokalisering en gedeeltelike karakterisering van nuklease-aktiwiteit in assies van Phaseolus vulgaris $L$. tydens ontkieming: 1,48 .

Van Rensburg, D.J.J. Technikons in die toekomstige SuidAfrika: 3, 144

Van Staden, J.F. Openingsrede - Simposium van die Afdeling Chemie oor Chemiese tegnologie in die toekomstige Suid-Afrika 11, 10 Augustus 1990: 3, 142.

Venter, A., H.J. Schoonbee en H.H. du Preez. 'n Ekologiese ondersoek na eutrofikasietoestande in die Floridameer, Roodepoort, met spesiale verwysing na die biologiese beheer van onderwatermakrofiete deur gebruik te maak van die Sjinese graskarp, Ctenopharyngodon idella (Vol.): 1, 55.

Visser, D.S., F. le R. Fourie en C. Huyser. In vitrokweking van muisembrio's in menslike amnionvog en Ham F10-medium: resultate en vergelykende biochemiese profiele van kweekmedia: 1, 61 .

Vosloo, H.C.M. en J.A.K. du Plessis. Die metatese van alkyne: 2,72 .

Wepener, V., J.H.J. van Vuren en H.H. du Preez. Die invloed van mangaanbesoedeling op die hematologie en koolhidraatmetabolisme van Tilapia sparrmanii (Cichlidae): 1, 53. 